University of Nebraska - Lincoln

DigitalCommons@University of Nebraska - Lincoln

$6-5-2000$

\title{
Electron Acceleration and the Propagation of Ultrashort High- Intensity Laser Pulses in Plasmas
}

\author{
Xiaofang Wang \\ University of Michigan, Ann Arbor \\ Mohan Krishnan \\ University of Michigan, Ann Arbor \\ Ned Saleh \\ University of Michigan, Ann Arbor \\ Haiwen Wang \\ University of Michigan, Ann Arbor \\ Donald P. Umstadter \\ University of Nebraska-Lincoln, donald.umstadter@unl.edu
}

Follow this and additional works at: https://digitalcommons.unl.edu/physicsumstadter

Part of the Physics Commons

Wang, Xiaofang; Krishnan, Mohan; Saleh, Ned; Wang, Haiwen; and Umstadter, Donald P., "Electron Acceleration and the Propagation of Ultrashort High-Intensity Laser Pulses in Plasmas" (2000). Donald Umstadter Publications. 23.

https://digitalcommons.unl.edu/physicsumstadter/23

This Article is brought to you for free and open access by the Research Papers in Physics and Astronomy at DigitalCommons@University of Nebraska - Lincoln. It has been accepted for inclusion in Donald Umstadter Publications by an authorized administrator of DigitalCommons@University of Nebraska - Lincoln. 


\title{
Electron Acceleration and the Propagation of Ultrashort High-Intensity Laser Pulses in Plasmas
}

\author{
Xiaofang Wang, Mohan Krishnan, Ned Saleh, Haiwen Wang, and Donald Umstadter \\ Center for Ultrafast Optical Science, University of Michigan, Ann Arbor, Michigan 48109-2099
}

(Received 4 February 2000)

\begin{abstract}
Reported are interactions of high-intensity laser pulses $\left(\lambda=810 \mathrm{~nm}\right.$ and $\left.I \leq 3 \times 10^{18} \mathrm{~W} / \mathrm{cm}^{2}\right)$ with plasmas in a new parameter regime, in which the pulse duration $(\tau=29 \mathrm{fs})$ corresponds to 0.6-2.6 plasma periods. Relativistic filamentation is observed to cause laser-beam breakup and scattering of the beam out of the vacuum propagation angle. A beam of megaelectronvolt electrons with divergence angle as small as $1^{\circ}$ is generated in the forward direction, which is correlated to the growth of the relativistic filamentation. Raman scattering, however, is found to be much less than previous longpulse results.

PACS numbers: 42.65.Jx, 52.35.Mw, 52.40.Nk
\end{abstract}

The propagation of high-intensity light pulses in plasmas is of fundamental interest and essential to technological applications such as electron accelerators, $\mathrm{x}$-ray lasers and fast-ignitor thermonuclear fusion [1]. Research into this problem has been possible only within the past few years, with the development of lasers capable of delivering pulses with field strengths sufficient to drive electrons to relativistic velocities [2]. In this regime, the effects of relativistic quiver motion as well as laser pressure dominate the interaction, causing, among other things, relativistic self-focusing (RSF) and electron acceleration [3].

The RSF threshold is given theoretically by a critical laser power [4], $P_{c}[\mathrm{GW}]=17\left(\omega_{0}^{2} / \omega_{p}^{2}\right)$, where $\omega_{0}$ is the laser frequency, and $\omega_{p}=\sqrt{n_{e} e^{2} / m \varepsilon_{0} \gamma}$ is the plasma frequency ( $m$ is electron mass, $n_{e}$ is the plasma electron density, $\gamma=\sqrt{1+a_{0}^{2} / 2}$ is the relativistic factor for a linearly polarized laser, and $a_{0}=8.5 \times 10^{-10} \lambda[\mu \mathrm{m}] \times$ $I^{1 / 2}\left[\mathrm{~W} / \mathrm{cm}^{2}\right]$ is the normalized laser intensity). Theory shows that, depending on the initial laser and plasma conditions, either whole-beam RSF (associated with optical guiding or self-channeling) or relativistic filamentation (a partial beam analog to the whole beam effect, or multiple filaments) can occur [5,6]. However, neither relativistic filamentation (as a mechanism of laser-beam breakup [7]) nor its correlation to the generation of megaelectronvolt $(\mathrm{MeV})$ electrons has been given much experimental attention. In fact, there seem to be divergent interpretations of the few experiments that have been reported on this topic; with similar laser and plasma-density conditions, relativistic filamentation was suggested in one case [7], while relativistic channeling was claimed in another $[8,9]$.

Generally, these previous experiments have used laser pulses with ratios of their longitudinal to transverse spatial extents much greater than unity. The transverse ponderomotive force should be dominant, which means that the interaction is multidimensional and transverse effects such as Raman sidescattering are important. Also, because there are a number of plasma periods $\left(\tau_{p}=2 \pi / \omega_{p}\right)$ within the pulse envelope, various instabilities such as Raman processes and laser-envelope modulations can cause laser beam breakup $[10,11]$ and complicate the interpretation of the origin of electron acceleration [9,12-14]. For instance, a recent analysis shows that the occurrence of Raman scattering and plasma heating will suppress both ponderomotive expulsion and RSF [15].

For ultrashort high-intensity pulses $\left(\tau \sim \tau_{p}\right)$, the physics may be significantly different and simpler, in that Raman scattering may not be important [15-17]. However, no experimental results have yet been presented, due to the difficulty in producing high-intensity pulses with such short pulse durations. Reported in this Letter are results on laser propagation that - to our knowledge - are the first in this regime, in which the laser pulse extent is close to, or shorter than, both the laser focal spot size and a plasma period. These are the same parameters required for many recently proposed laser-wakefield-accelerator concepts [18].

The experiments were performed with a tabletop multiterawatt, ultrashort Ti:sapphire laser that delivered $29 \mathrm{fs}$ pulses at $810 \mathrm{~nm}$, with repetition rate $10 \mathrm{~Hz}$ [19]. The intensity contrast was measured $\sim 10^{-5}$ on the nanosecond scale. High-dynamic-range pulsewidth measurements showed that the laser pulse shape was well approximated by a Gaussian temporal profile of $\tau=29$ fs in full width at half maximum (FWHM). The pulse contained 93\% of the laser energy in the range from the Gaussian pulse peak to \pm 38 fs, beyond which there were wings, extending to $\pm 200 \mathrm{fs}$, with less than $7 \%$ of the laser energy. The wings were due to incomplete compensation by the grating compressor, resulting in an intensity contrast better than $10^{-5}$ at \pm 200 fs. The horizontally linear-polarized, 4-cm diameter laser beam was focused with an $f / 4.5$, $45^{\circ}$ off-axis parabolic mirror onto a supersonic gas jet. The vacuum spot [see Fig. 2 (below)] size was $r_{0}=$ $8 \mu \mathrm{m}$ (radius in $1 / e^{2}$ intensity) or the Rayleigh range was $X_{R}=250 \mu \mathrm{m}$, containing $50 \%$ of laser energy. For incident laser power $P=6 \mathrm{TW}$, this led to peak intensity $I=3 \times 10^{18} \mathrm{~W} / \mathrm{cm}^{2}$ or $a_{0}=1.2$. The supersonic gas jet, which forms a 750- $\mu \mathrm{m}$ diameter, sharp-edged flat-top-profile gas plume, was backed by $N_{2}$ to produce the required plasma electron density, up to $10^{20} / \mathrm{cm}^{3}$. The 
plasma density was calibrated with Raman backscatter by a long pulse at low intensity $\left(\sim 10^{17} \mathrm{~W} / \mathrm{cm}^{2}\right)$ and the density fluctuation ranged from $\pm 20 \%$ at $n_{e}=1 \times 10^{20} / \mathrm{cm}^{3}$ to $\pm 40 \%$ at $n_{e}=6 \times 10^{18} / \mathrm{cm}^{3}$. A barrier-suppression ionization model [20] indicates that a stable ionization stage or plasma electron density is obtained because nitrogen is ionized to He-like ionization states above a laser intensity of $1.4 \times 10^{16} \mathrm{~W} / \mathrm{cm}^{2}$ and below $1.0 \times$ $10^{19} \mathrm{~W} / \mathrm{cm}^{2}$. Thus ionization-induced defocusing should not be significant and RSF should be obtainable [21].

Light that is transmitted through the underdense plasma was collected by a lens with an $f / 3.4$ acceptance aperture to an energy calorimeter. The transmission measurement is shown in Fig. 1. For convenience, we used a plasma electron density normalized to the critical density $n_{c}\left(\omega_{0}=\right.$ $\left.\omega_{p}\right)$, which for $810 \mathrm{~nm}$ equals $1.7 \times 10^{21} / \mathrm{cm}^{3}$. As shown in Fig. 1(a), at low powers, the transmitted energy is close to the incident energy. (For such plasma densities, there is $\sim 1 \%$ of laser energy loss to ionization of the neutral gas.) With increasing laser power, the transmission loss becomes larger. Varying the plasma density changes the laser power at which the inflection points occur from nearly $100 \%$ transmission to evident transmission loss. We have found that the transmitted energy scales linearly to the incident laser power [see dotted lines in Fig. 1(a)], and in this way we can get the inflection point from the intersection between the $100 \%$ transmission line and the measured transmission line. Similar scaling phenomena can be found in Ref. [7]. For $n_{e}=0.015 n_{c}\left(0.06 n_{c}\right)$, the inflection occurs at $P=0.84 \mathrm{TW}(0.28 \mathrm{TW})$. Note that the power is very close to $1.1 \mathrm{TW}(0.28 \mathrm{TW})$, the predicted $P_{c}$ for RSF. By fixing the incident laser power at $6 \mathrm{TW}$ and varying the plasma density, it is found that the point at which transmission loss becomes apparent is around $n_{e}=0.015 n_{c}$ [see Fig. 1(b)], for which density the laser power $P \approx$ $5 P_{c}$. These results are consistent with transmission loss caused by the onset of RSF and the growth of relativistic filamentation.

When relativistic filamentation appears, the laser is predicted to be focused to a spot size $\sim c / \omega_{p}$. For a Gaussian beam this means that the diffraction angle of the laser beam will increase. To verify this, we increased the accep-
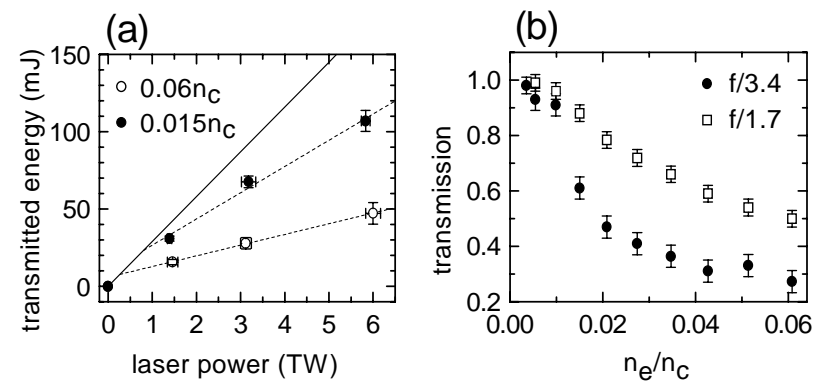

FIG. 1. (a) Energy transmission as a function of incident laser power. The solid line corresponds to $100 \%$ transmission. (b) Energy transmission as a function of plasma density for an incident laser power of $6 \mathrm{TW}$. tance angle of the collecting lens to $f / 1.7$ [see Fig. 1(b)]. Comparison of the measurements taken at the two collection angles shows that at around $n_{e}=0.015 n_{c}$ the scatter becomes evident. The spectra of the sidescattered light indicate that most of the scattered light is from the laser fundamental and the redshifted spectral component is negligible.

The light transmitted through the plasma passed through an optical-quality, dielectric-coated mirror, which reflected four orders of the fundamental light at $810 \mathrm{~nm}$ and was imaged with an $f / 4$ achromatic lens that formed a magnified image at a 16-bit charge-coupled device (CCD) with a resolution of about $4 \mu \mathrm{m}$. An $810-\mathrm{nm}$ bandpass filter with $\pm 5 \mathrm{~nm}$ bandwidth was put before the CCD to define the spectral range of the image. A sequence of the images for different plasma densities is shown in Fig. 2, which give the equivalent-plane image of the laser beam at a position $400 \mu \mathrm{m}$ (or $1.6 X_{R}$ ) past the best vacuum focal position. Beyond this position, we found that the laser beam scattered quickly. Nevertheless, the laser beam could be focused as well as it could in vacuum when the equivalent-plane-image position was close to the best vacuum focal position. From Fig. 2, it can be seen that at low plasma densities the laser beam is defocused. At higher plasma densities, part of the beam is self-focused and the low-intensity part is defocused. Around $n_{e}=0.03 n_{c}$, there appear two filaments surrounded by scattered light, and at higher densities the scattered light increases, indicating beam breakup. The apparent size of these filaments (4-5 $\mu \mathrm{m}$ FWHM) is smaller than the vacuum focal size (9 $\mu \mathrm{m}$ FWHM).

The observed filament size may be compared with relativistic filamentation predicted in Ref. [5]. The predicted filament size is given by $4 \pi c / \omega_{p} a_{0}$, which for $n_{e}=0.05 n_{c}$ and $a_{0}=1.2$ gives $6 \mu \mathrm{m}$, close to the measurements. Note that increasing either the laser intensity (through RSF) or the plasma density can cause larger growth of the relativistic filamentation and further reduction of the filament size, consistent with the condition for relativistic filamentation, $4 \pi c / \omega_{p} a_{0} \approx 2 r_{0}$, where " " applies to the special case RSF. Particle-in-cell simulations [22] indicate that for higher laser intensities $\left(>10^{19} \mathrm{~W} / \mathrm{cm}^{2}\right)$ the filament size could be $1-2$ times the laser wavelength.

Along with the growth of relativistic filamentation, $\mathrm{MeV}$ electrons are observed in the forward direction. For this measurement a scintillator screen, LANEX, imaged by a 16-bit CCD, was placed in the forward direction of the laser beam, to provide the angular distribution of the electron beam [23]. A plastic scintillator coupled to a photomultiplier tube (S/PMT, which was calibrated with a radioactive source), read out by an oscilloscope, was placed outside the vacuum chamber window of enhanced Mylar foil, to record, in the forward direction, the number of electrons. The S/PMT measurement exhibits two features: (i) for $P=6 \mathrm{TW}$, the electron signal first appears at $n_{e}=0.015 n_{c}$, which corresponds to $\tau / \tau_{p}=1.32$, 

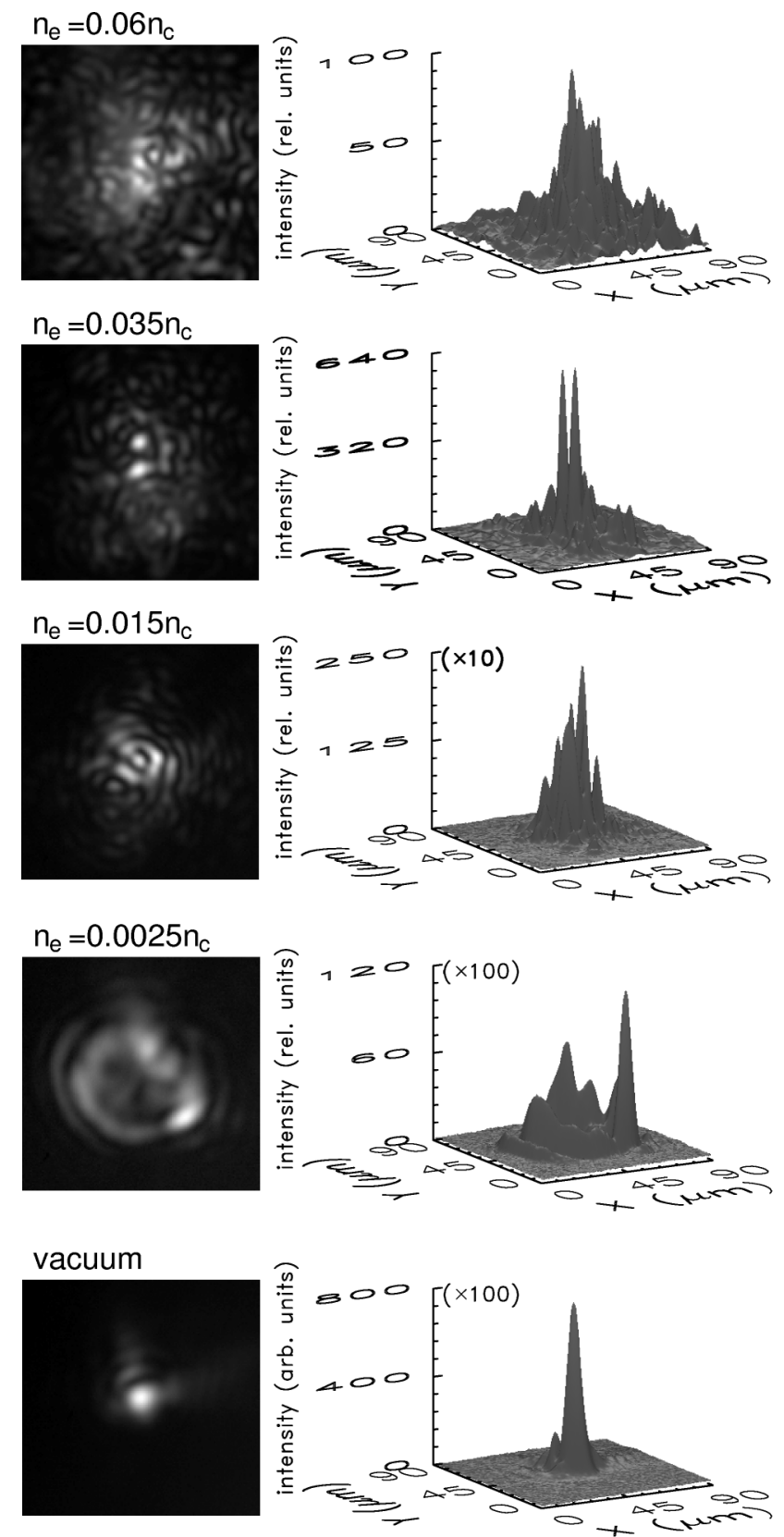

FIG. 2. Microscopic images of the transmitted light as a function of plasma density and the corresponding intensity distributions for an incident laser power of $6 \mathrm{TW}$. The image labeled "vacuum" is the laser beam image at the best focal position under vacuum and the other images correspond to $400 \mu \mathrm{m}$ past the best vacuum focal position.

and increases with increasing plasma density. There is no electron signal at lower plasma densities. Note that at this density there appears an evident reduction of the transmitted laser light (see Fig. 1). These observations indicate a correlation between relativistic filamentation and the electron signal. (ii) For $n_{e}=0.06 n_{c}$, the electron signal first appears at laser power $P \approx 2 \mathrm{TW}$, and increases with increasing laser power. For either density, electrons are generated only when $P \geq 5 P_{c}$.
The LANEX measurement shows that the electrons are emitted as a collimated beam in the forward direction. The full angular spread of the beam is $\sim 15^{\circ}$. However, there is a main beam component in the whole beam, which has a divergence angle of only $1^{\circ}$ FWHM, as shown in Figs. 3(a) and 3(c). Since we have not seen evidence of whole-beam optical guiding, which has previously been found to be correlated with a decrease in the divergence of electron beam [23], it appears that these electrons in the main beam are generated instead from a dominant filament. This is in agreement with Refs. [12,14,22], in which numerical simulations are reported, showing that electrons accelerated in a filament can have high energies and small angular spreads. In combination with the S/PMT measurement, we estimate that, for $n_{e}=0.06 n_{c}$, the number of electrons with kinetic energies above $1 \mathrm{MeV}$ is about $10^{5}$, and the number of electrons in the main beam component is $\sim 5 \times 10^{4}$.

The energy spectrum of the electrons, passing through a 2.4-degree-aperture collimator in the direct forward, was measured by use of a magnetic spectrometer. The result is shown in Fig. 3(d), which is an average of a number of shots with similar laser and plasma conditions. At low electron kinetic energy, the number is underestimated because of the nonlinear dispersion of the spectrometer. It is found that the number of electrons versus energy fits an exponential decay $d N / d E \propto \exp \left(-E / T_{e}\right)$, with temperature $T_{e}=1.1 \mathrm{MeV}$. Note the temperature is higher than that in Ref. [23] where optical guiding was suggested for the enhancement of electron acceleration. (a)
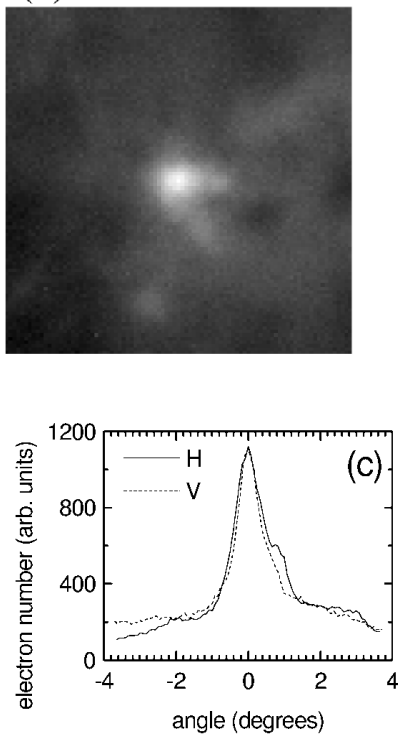
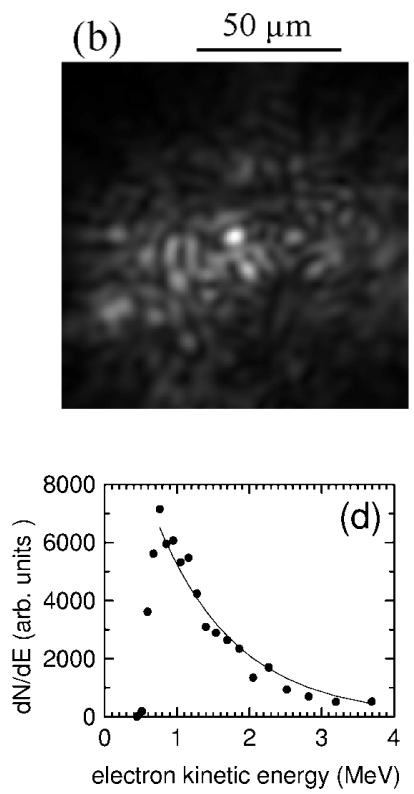

FIG. 3. LANEX image of the main electron-beam component (a) and lineouts in the horizontal $(\mathrm{H})$ and vertical $(\mathrm{V})$ directions through the beam center (c). (b) Microscopic image of the redshifted component of the transmitted light. (d) Measured electron number distribution versus energy (points) and exponential-decay fit (solid line). The incident laser power is 6 TW and the plasma density is $n_{e}=0.06 n_{c}$. 
Spectra in the backward, sideward, and forward directions were measured with a prism spectrometer, recorded by an 18-bit CCD, with a spectral resolution of $\Delta \lambda=$ $3 \mathrm{~nm}$. For sidescatter, the light was collected at $34^{\circ}$ from the laser axis with an $f / 7$ collection angle. As mentioned above, we found that the sidescattering is negligible. For backscattering, the light was collected with the same parabola for the plasma creation, and the measured signal was weak but with typical Raman backscatter features (a redshifted component) when the laser power was greater than $3 \mathrm{TW}$. By integrating the redshifted spectra from $850-1200 \mathrm{~nm}$, we measured that for $P=6 \mathrm{TW}$ the fraction of the backscattered light was smaller than $10^{-4}$ of the incident laser energy at $n_{e}=0.06 n_{c}$, which is much less than previous measurements with longer pulses [24].

The forward-scattered light was collected with the same imaging optics for the transmitted light. The main feature observed was that the redshifted spectra showed a broad bandwidth $(\sim 80 \mathrm{~nm}$ FWHM), with spectral peak positions extending from $\sim 900 \mathrm{~nm}\left(n_{e}=0.01 n_{c}\right)$ to $\sim 1000 \mathrm{~nm}\left(n_{e}=0.06 n_{c}\right)$. No higher order peaks were observed. For lower plasma densities $\left(n_{e}<0.015 n_{c}\right)$, even when no $\mathrm{MeV}$ electrons were detected, the redshift was still observed. For the forward Raman-scatter instability, the amount of gain $\left(e^{G}\right)$ should be given by [11] $G=2 \Gamma_{\mathrm{rfs}} \sqrt{\left(X_{R} / c-\Delta t_{L}\right) \Delta t_{L}}$ and $\Gamma_{\mathrm{rfs}}=\left(n_{e} / n_{c}\right) \times$ $\left(a_{0} / \sqrt{8} \gamma^{2}\right) \omega_{0}$. For $n_{e} / n_{c}=0.015$ (0.03), $G=2.6$ (5.2), indicating that Raman forward scattering should be insignificant. Moreover, the laser bandwidth $\Delta \omega_{L}=9 \times$ $10^{13} / \mathrm{s}$ is much larger than the Raman instability growth rate $\left[\Gamma_{\mathrm{rfs}} / \Delta \omega_{L}=0.1(0.2)\right.$ for $n_{e} / n_{c}=0.015(0.03)$, respectively] and can also act to suppress the Raman process [25]. The redshifting might be caused by either (or a combination of) (i) electron expulsion by relativistic filamentation [7], (ii) frequency downshift or photon deceleration due to the depletion of laser energy in driving a wakefield [16], or (iii) relativistic self-modulation [5] and self-phase modulation (the index of refraction is $\left.\sqrt{1-\omega_{p}(I(t), t)^{2} / \omega_{0}^{2}}\right)$ due to the relativistic increase of electron mass.

Using the same imaging measurement for the transmitted light but with an IR bandpass filter in front of CCD, the image of the redshifted component at $400 \mu \mathrm{m}$ past the vacuum focus was measured. A typical image is shown in Fig. 3(b), which shows a similar structure to that of the laser fundamental (refer to Fig. 2), which suggests that the largest contribution to the redshifted component is filamentation. This seems consistent with the correlation observed between the $\mathrm{MeV}$ electrons and filamentation. Actually the $\mathrm{MeV}$ electron signal was also correlated with the redshifted spectral brightness. The redshifted spectral image was $\sim 3 \times$ brighter with an electron signal than without it for the same incident energy. In either case, the fraction of the redshifted component was measured to be less than $2 \%$ of the incident laser energy, which indicates that the transmitted light from the plasma (see Fig. 1) is mainly the same frequency as the laser fundamental.

We acknowledge the support of the High-Energy Physics Division of the U.S. Department of Energy with laser facilities supported by the National Science Foundation. We also thank S. Backus, H. Kapteyn, and M. Murnane for their contributions to the laser system.

[1] G. Mourou et al., Phys. Today 51, No. 1, 22 (1998).

[2] Feature Issue on Optics of Relativistic Electrons, edited by D. Umstadter and T. Norris [IEEE J. Quantum Electron. 33, 1877 (1997)].

[3] E. Esarey et al., IEEE J. Quantum Electron. 33, 1879 (1997), and references therein.

[4] P. Sprangle et al., IEEE Trans. Plasma Sci. 15, 145 (1987); G. Z. Sun et al., Phys. Fluids 30, 526 (1987).

[5] C. Max et al., Phys. Rev. Lett. 33, 209 (1974).

[6] A. B. Borisov et al., Plasma Phys. Controlled Fusion 37, 569 (1995); F. Vidal and T. W. Johnston, Phys. Rev. Lett. 77, 1282 (1996); M. D. Feit et al., Phys. Rev. E 57, 7122 (1998).

[7] P. E. Young and P. R. Bolton, Phys. Rev. Lett. 77, 4556 (1996).

[8] M. Borghesi et al., Phys. Rev. Lett. 78, 879 (1997).

[9] C. Gahn et al., Phys. Rev. Lett. 83, 4772 (1999).

[10] P. Sprangle et al., Phys. Rev. Lett. 69, 2200 (1992); T. M. Antonsen, Jr. and P. Mora, Phys. Rev. Lett. 69, 2204 (1992).

[11] W. B. Mori et al., Phys. Rev. Lett. 72, 1482 (1994).

[12] J. C. Adam et al., Phys. Rev. Lett. 78, 4765 (1997).

[13] K.-C. Tzeng et al., Phys. Rev. Lett. 79, 5258 (1997); E. Esarey et al., Phys. Rev. Lett. 80, 5552 (1998).

[14] A. Pukhov et al., Phys. Plasmas 6, 2847 (1999).

[15] K.-C. Tzeng and W. Mori, Phys. Rev. Lett. 81, 104 (1998).

[16] C. D. Decker et al., Phys. Plasmas 3, 2047 (1996).

[17] P. Mora and T. M. Antonsen, Jr., Phys. Rev. E 53, R2068 (1996).

[18] D. Umstadter et al., Phys. Rev. Lett. 76, 2073 (1996); E. Esarey et al., Phys. Rev. Lett. 79, 2682 (1997).

[19] H. Wang et al., J. Opt. Soc. Am. B 16, 1790 (1999).

[20] S. Augst et al., Phys. Rev. Lett. 63, 2212 (1989).

[21] R. Fedosejevs et al., Phys. Rev. E 56, 4615 (1997).

[22] A. Pukhov and J. Meyer-ter-vehn, Phys. Rev. Lett. 76, 3975 (1996).

[23] R. Wagner et al., Phys. Rev. Lett. 78, 3125 (1997).

[24] X.F. Wang et al., Opt. Commun. 146, 363 (1998); C. Rousseaux et al., Phys. Rev. Lett. 74, 4655 (1995).

[25] J. J. Thomson and J. I. Karush, Phys. Fluids 17, 1608 (1974). 